\title{
A HARDY INEQUALITY FOR THE GRUSHIN TYPE OPERATORS
}

\section{ZHU}

Abstract. We prove a Hardy inequality related to Carnot-Carathéodory distance for the Grushin type operators like $\Delta_{x}+|x|^{2} \partial_{t}^{2}$. The procedure is based on a representation formula for such operators.

Mathematics subject classification (2010): Primary 26D10; 35H20.

Keywords and phrases: Hardy inequality, Grushin type operators, Carnot-Carathéodory distance.

\section{REFERENCES}

[1] W. CoHn, G. LU, Best constants for Moser-Trudinger inequalities on the Heisenberg group, Indiana Univ. Math. J. 50 (2001), 1567-1591.

[2] L. D'A mbrosio, Some Hardy Inequalities on the Heisenberg Group, Differential Equations 40 (2004), $552-564$.

[3] L. D’Ambrosio, Hardy inequalities related to Grushin type operators, Proc. Amer. Math. Soc. 132 (2004), 725-734.

[4] L. D’ Ambrosio, Hardy-type inequalities related to degenerate elliptic differential operators, Ann. Sc. Norm. Super. Pisa Cl. Sci. (5) IV (2005), 451-486.

[5] N. Garofalo, E. LANCONELli, Frequency functions on the Heisenberg group the uncertainty principle and unique continuation, Ann. Inst. Fourier (Grenoble) 40 (1990), 313-356.

[6] P. C. Greiner, D. Holcman, Y. Kannai, Wave kernels related to second-order operators, Duke Math. J. 114, 2 (2002), 329-386.

[7] R. Monti, Some properties of Carnot-Carathéodory balls in the Heisenberg group, Atti Accad. Naz. Lincei Cl. Sci. Fis. Mat. Natur. Rend. Lincei (9) Mat. Appl. 11, 3 (2000), 155-167.

[8] R. Monti, D. Morbidelli, The isoperimetric inequality in the Grushin plane, J. Geom. Anal. 14, 2 (2004), 355-368.

[9] R. Monti, F. Serra Cass ano, Surface measures in Carnot-Carathéodory spaces, Calc. Var. Partial Differential Equations 13, 3 (2001), 339-376.

[10] P. NiU, H. Zhang, Y. WAng, Hardy type and Rellich type inequalities on the Heisenberg group, Proc. Amer. Math. Soc. 129 (2001), 3623-3630.

[11] M. PAUlat, Heat kernel estimates for the Grusin operator, arXiv:0707.4576v1 [math.AP].

[12] Q-H. YANG, Hardy type inequalities related to Carnot-Carathéodory distance on the Heisenberg group, preprint. 